\title{
Expression of the lysyl oxidase propeptide in hepatocellular carcinoma and its clinical relevance
}

\author{
YING ZHENG $^{1^{*}}$, XUEMEI WANG ${ }^{2 *}$, HAIDONG WANG ${ }^{3}$, WEI YAN ${ }^{4}$, QUAN ZHANG $^{5}$ and XIN CHANG \\ ${ }^{1}$ Department of Anesthesia, Huai'an First People's Hospital, Nanjing Medical University, Huai'an, Jiangsu 223300; \\ ${ }^{2}$ Department of Ultrasound, Obstetrics and Gynecology Hospital of Fudan University, Shanghai 200011; \\ Departments of ${ }^{3}$ Obstetrics and Gynecology, ${ }^{4}$ Gastroenterology, ${ }^{5}$ Oncology, and ${ }^{6}$ Imaging Medicine Center, \\ Huai'an First People's Hospital, Nanjing Medical University, Huai'an, Jiangsu 223300, P.R. China
}

Received December 24, 2013; Accepted January 20, 2014

DOI: $10.3892 /$ or.2014.3044

\begin{abstract}
Lysyl oxidase is an important extracellular matrix remodeling enzyme and plays critical roles in tumor progression and development. Its tumor-suppressor activity has been shown to depend on the propeptide region. Previous studies have reported that the expression levels of lysyl oxidase propeptide (LOX-PP) are associated with cancer of the breast, pancreas, lung, prostate and gastrointestinal system. However, to date, the exact effects and molecular mechanisms of LOX-PP in hepatocellular carcinoma progression are still unclear. The present study aimed to investigate the expression and clinical significance of LOX-PP in human hepatocellular carcinoma. First, 42 cases of hepatocellular carcinoma and corresponding adjacent non-cancerous tissues (ANCTs) were collected, and the expression of LOX-PP in these samples was assessed by immunohistochemistry (IHC). The clinicopathological characteristics of all patients were recorded. Next, in in vitro studies, recombinant adenovirus LOX (ad-LOX-PP) was used to infect hepatocellular carcinoma cell lines to determine the function of LOX-PP. To determine whether ad-LOX-PP affects hepatocellular carcinoma cell survival, cell viability was examined by CCK-8 assay, and cell cycle progression was assessed by flow cytometry. We also investigated the effects of LOX-PP on the expression of cell cycle regulators (cyclin D1 and cyclin E) by western blot analysis. The migration and invasion capacities of hepatocellular carcinoma cells were observed by wound-healing and Tranwell invasion assays. To further investigate how LOX-PP affects migration levels of matrix metallopeptidase (MMP)-2 and MMP-9 were assessed by western blot analysis. Additionally, markers of the PI3K and
\end{abstract}

Correspondence to: Dr Xin Chang, Department of Imaging Medicine Center, Huai'an First People's Hospital, Nanjing Medical University, 6 Beijing Road West, Huai'an, Jiangsu 223300, P.R. China E-mail: changxin317@126.com

${ }^{*}$ Contributed equally

Key words: lysyl oxidase propeptide, hepatocellular carcinoma, growth, migration, extracellular signal-regulated kinase
MAPK signaling pathway were detected to further confirm the mechanisms of LOX-PP. As a result, reduced expression of LOX-PP was found in hepatocellular carcinoma tissues, when compared with that in the ANCTs ( 15 vs. $83 \%, \mathrm{P}<0.01)$, and its expression was associated with tumor stage and distant metastasis (each $\mathrm{P}<0.05)$. Proliferation in hepatocellular carcinoma cells was significantly decreased in the ad-LOX-PP group as indicated by CCK-8 assay. LOX-PP significantly reduced the expression of $\mathrm{Ki}-67$, while prominent increases in the rate of apoptosis and cell cycle arrest were observed. Similarly, cell migration was significantly inhibited in the ad-LOX-PP group as evidenced by Transwell invasion and wound-healing assays. The expression levels of MMP-2 and MMP-9 were attenuated in the ad-LOX-PP group, suggesting that LOX-PP inhibits hepatocellular carcinoma cell migration via down-regulation of MMPs expression. When LOX-PP expression was potentiated by an adenovirus containing LOX-PP, the expression of p-ERK was significantly downregulated, indicating that LOX-PP inhibits hepatocellular carcinoma cell proliferation and induces its apoptosis probably through downregulation of the MAPK/ERK pathway.

\section{Introduction}

Hepatocellular carcinoma is the most common fatal malignancy due to the prevalence of hepatitis infection (hepatitis $B$ or C) (1). In spite of an increased understanding of hepatocarcinogenesis $(2,3)$, the molecular pathogenesis of hepatocellular carcinoma remains unclear (4). Therefore, it is necessary to explore the molecular signaling mechanism of hepatocellular carcinoma development to design novel therapeutic agents.

Lysyl oxidase (LOX), originally named the ras recision gene, is a key enzyme required for the normal biosynthesis of collagen and elastin maturation $(5,6)$. LOX is processed to a functional 32-kDa LOX enzyme and an $18-\mathrm{kDa}$ LOX propeptide (LOX-PP). Recent evidence has emerged for the double-edged role of LOX as both a tumor-suppressor and a promoter of metastasis, while LOX-PP has definite tumorsuppressive effects (7-9). LOX-PP is expressed by various cell types, and its low expression level is correlated with several tumor types (7-9). In vitro studies have shown that LOX-PP reverses the invasive phenotype of Her2/neu breast cancer 
cells, and inhibits the migration and branching colony formation (10). LOX-PP was also found to inhibit RAS-dependent transformation of NIH $3 \mathrm{~T} 3$ fibroblasts (11). In addition, LOX-PP promoted the apoptosis induced by chemotherapeutic drugs (12). In vivo, injection of LOX-PP significantly reduced the tumor volume and the expression of proliferation markers in a mouse xenograft model (7). Retrovirus-mediated ectopic overexpression of LOX-PP suppressed xenograft tumor formation in nude mice (10). However, the exact role of LOX-PP in hepatocellular carcinoma has not been investigated (13).

We, therefore, investigated the expression and clinical significance of LOX-PP in hepatocellular carcinoma tissues. We infected hepatocellular carcinoma cell lines with the recombinant adenovirus LOX-PP (ad-LOX-PP) to determine the relative antitumor function of LOX-PP. We then assessed the expression levels of markers which are related to proliferative activities and invasive potential, and cell apoptosis and cell cycle distribution.

\section{Materials and methods}

Samples. All experimental procedures were carried out at the First People's Hospital of Huai'an and approved by the Hospital Ethics Committee. From 2009 to 2011, primary hepatocellular carcinoma surgically resected from 42 patients who received detailed pathological assessment and a regular 2-year follow-up were selected for the present study. All of the specimens were anonymized and evaluated in a blinded manner. The patients included 25 men and 17 women with a mean age of 50.6 years (range, 35-68). After surgery, all patients received laboratory examinations such as serum $\alpha$-fetoprotein (AFP) and ultrasonography of the liver at 3-6 month intervals. Surgically resected specimens were formalin-fixed, paraffin-embedded and cut into 5- $\mu \mathrm{m}$ sections. The sections were stained with hematoxylin and eosin (H\&E) and reviewed by one of the authors (X.C.) to determine tumor grade and stage as proposed by Edmonson and Steiner (14). The margins of the surgical specimens were inked and checked under a microscope.

Reagents. An apoptosis detection kit (Annexin V-PE/PI), the Cell Counting Kit-8 (CCK-8) and the ECL-Plus kit was purchased from Beyotime Institute of Biotechnology (Haimen, China). Dulbecco's modified Eagle's medium(DMEM) and fetal bovine serum (FBS) were purchased from Gibco (Montevideo, Uruguay). M-MLV reverse transcriptase was purchased from Promega (Madison, WI, USA), and Lipofectamine 2000 was purchased from Invitrogen (Carlsbad, CA, USA).

Cell culture. A poorly differentiated (SK-Hep-1) and a welldifferentiated (HepG2) human hepatocellular carcinoma cell line were purchased from the Shanghai Cell Research Institution of the Chinese Academy of Science. Human hepatocellular carcinoma cell lines were cultured in DMEM supplemented with $10 \% \mathrm{FBS}$, and antibiotics (100 IU/ml of penicillin $\mathrm{G}$ and $100 \mathrm{ng} / \mathrm{ml}$ of streptomycin). They were all incubated at $37^{\circ} \mathrm{C}$ in a humidified atmosphere of $5 \% \mathrm{CO}_{2}$, and the medium was replaced every 2 days.

Cell proliferation assay. Cell proliferation was analyzed using the CCK- 8 assay. Briefly, the cells were incubated in 96-well plates at a density of $0.5 \times 10^{5}$ cells/well. Following recovery of transfection for $48 \mathrm{~h}$, cells were cultured at 6, 12, 24, 48, 72 and $96 \mathrm{~h}$ and then incubated with $10 \mu \mathrm{l} \mathrm{CCK}-8$ dye for $30 \mathrm{~min}$ at $37^{\circ} \mathrm{C}$. The absorbance of optical densities was measured at $550 \mathrm{~nm}$ with an enzyme immunoassay analyzer (Bio-Rad Laboratories, Hercules, CA, USA).

Measurement of apoptosis by flow cytometry. The rate of apoptosis was detected using the Annexin V-PE/PI apoptosis detection kit (Invitrogen) according to the method of Yang et al (15). Apoptotic cells, including those staining positive for Annexin V-PE and negative for PI and those that were double positive, were counted.

Cell cycle analysis. For cell cycle analysis, harvested cells were centrifuged at $1000 \mathrm{x}$ g for $5 \mathrm{~min}$ and fixed in $70 \%$ ethanol overnight. Cells were then washed with PBS, re-suspended and treated with RNase $(10 \mu \mathrm{g} / \mathrm{ml})$ for $30 \mathrm{~min}$ at $37^{\circ} \mathrm{C}$. Subsequently, cells were stained in $1 \mathrm{ml}$ of $50 \mu \mathrm{M}$ propidium iodide (PI) for $30 \mathrm{~min}$. The cell cycle phase distribution was determined by analytical DNA flow cytometry as described by Chiu et al (16). The percentage of cells in each phase of the cell cycle was analyzed using ModFit software (Verity Software House, Topsham, ME, USA).

Adenovirus transfection. Recombinant adenovirus vector ad-LOX-PP and negative control ad-GFP were transfected into the SK-Hep-1 cells. In preliminary experiments, cells were co-infected with recombinant adenovirus vector LOX-PP (ad-LOX-PP group) or negative control ad-GFP (ad-GFP group) at MOIs of 80-100 pfu/cell, according to the manufacturer's instructions. Cells were subcultured at a 1:3 dilution in $400 \mu \mathrm{g} / \mathrm{ml} \mathrm{G} 418$-containing medium to select the cells with stable transfectants. Subsequently, followed by a 48-h recovery after transfection, proliferation, metastasis and invasion were assessed in the ad-LOX-PP group, ad-GFP group and control group (SK-Hep-1 cell without gene transfection), respectively.

Immunohistochemistry. The levels of LOX-PP protein expression in the tumor tissues and the ANCTs were assessed by immunohistochemistry. The sections were then blocked in $3 \%$ goat serum (Invitrogen) for $1 \mathrm{~h}$ and incubated with the LOX-PP antibody (diluted 1:500) for $2 \mathrm{~h}$ at room temperature. A negative control was performed using a section without the primary antibody. Subsequently, antibody binding was visualized by incubation with fluorescent dye 4',6-diamidino2-phenylindole (DAPI) (Wuhan Boster Biological Technology, Ltd., Wuhan, China) for $3 \mathrm{~min}$ at room temperature. The LOX-PP immunoreactive and non-immunoreactive cells with a stained nucleus were counted in 5 sections (with an interval of 3 sections) per patient in each group. The positive staining of LOX-PP was shown as brown particles and the nucleus as blue. The sections were observed and photographed with an optical microscope (Olympus, Tokyo, Japan). The LOX-PP-positive cell percentage was calculated as the ratio of immunostained/ total nuclei x $100 \%$.

Western blot assay. The cells were extracted at indicated times using lysis buffer. Cell extracts were boiled for $10 \mathrm{~min}$ 
in loading buffer, and equal amounts of cell extracts were then separated on $10 \%$ SDS-PAGE gels. Separated protein bands were transferred to polyvinylidene fluoride membranes, and the membranes were blocked in 5\% skim milk powder. The primary antibodies against LOX-PP, AKT, p-AKT, ERK, p-ERK, PI3K, PI3K, JNK, p-JNK, P38, p-P38, Ki-67, MMP-2, MMP-9, cyclin E and cyclin D1 were diluted according to the instructions for the antibodies and incubated overnight at $4^{\circ} \mathrm{C}$. Subsequently, horseradish peroxidase-linked secondary antibodies were incubated at room temperature for $4 \mathrm{~h}$ at a dilution ratio of 1:1,000. The membranes were washed with TBST three times, and the immunoreactive bands were visualized using the ECL-Plus kit according to the instructions provided in the kit. The relative protein level in the different cell lines was normalized to the $\beta$-actin concentration.

Wound-healing assay. SK-Hep-1 cells were plated in each well of a 12-well culture plate and allowed to grow to $80 \%$ confluency. After recovery of transfection for $48 \mathrm{~h}$, a wound was created using a 10- $\mu 1$ micropipette tip. The migration of cells towards the wound was monitored at times 24 and $48 \mathrm{~h}$.

Transwell invasion assay. Briefly, the cells were trypsinized with $0.25 \%$ trypsin containing EDTA. Subsequently, cells were suspended and implanted with a Transwell insert with $8-\mu \mathrm{m}$ pore size coated with $50 \mu \mathrm{l}$ Matrigel (BD Biosciences), and $200 \mu \mathrm{l}$ medium containing $15 \%$ FBS was added in the bottom chamber. Following migration for $24 \mathrm{~h}$, a cotton swab was used to remove the non-migrated cells in the upper chamber, and then the filters were individually fixed with $4 \%$ paraformaldehyde. The cell numbers were counted in five random fields for each chamber under a microscope.

Statistical analysis. The data analyses were carried out using SPSS (SPSS 13.0 software). Correlation between LOX-PP expression and the clinicopathological parameters was evaluated using the $\chi^{2}$ test. Student's t-test was used for the comparisons between two groups and one-way analysis of variance (ANOVA) using Fisher's test for multiple comparisons. All data are presented as means \pm standard error (SE) for at least three independent experiments. Statistical significance was set at $\mathrm{P}<0.05$ (two-tailed).

\section{Results}

Expression of LOX-PP protein in hepatocellular carcinoma tissues and ANCTs. Expression of LOX-PP protein was reduced in tumors as evidenced by IHC assay. As shown in Fig. 1A, the expression of LOX-PP protein in hepatocellular carcinoma tissues was detected in 34 cases, while it was not found in the other 8 cases. The protein was detectable in all of the ANCTs. Furthermore, the mean positive cell incidence of LOX-PP protein staining was $83.3 \%$ in ANCTs, as compared with $15.0 \%$ in the hepatocellular carcinoma tissues with a significant difference $(\mathrm{t}=2.08 ; \mathrm{P}<0.001)$.

Correlation of LOX-PP protein expression with the clinical and pathological characteristics. The relationship between LOX-PP expression and various clinical and pathological features was analyzed. As shown in Table I, no significant
Table I. Correlation of LOX-PP expression and clinicopathological characteristics of the hepatocellular carcinoma cases.

\begin{tabular}{|c|c|c|c|c|c|}
\hline \multirow[b]{2}{*}{ Variables } & \multirow[b]{2}{*}{ Cases (n) } & \multicolumn{2}{|c|}{$\begin{array}{l}\text { LOX-PP } \\
\text { expression }\end{array}$} & \multirow[b]{2}{*}{$\chi^{2}$} & \multirow[b]{2}{*}{ P-value } \\
\hline & & - & + & & \\
\hline \multicolumn{6}{|c|}{ Age (years) } \\
\hline$<55$ & 28 & 5 & 23 & 1.075 & 0.299 \\
\hline$\geq 55$ & 14 & 3 & 11 & & \\
\hline \multicolumn{6}{|l|}{ Gender } \\
\hline Male & 25 & 4 & 21 & 0.554 & 0.541 \\
\hline Female & 17 & 4 & 13 & & \\
\hline \multicolumn{6}{|c|}{ AFP (ng/ml) } \\
\hline$<500$ & 19 & 3 & 16 & 1.230 & 0.267 \\
\hline$\geq 500$ & 23 & 7 & 16 & & \\
\hline \multicolumn{6}{|c|}{ Tumor stage } \\
\hline $\mathrm{I}$ & 11 & 7 & 4 & 19.216 & 0.001 \\
\hline II-III & 31 & 1 & 30 & & \\
\hline \multicolumn{6}{|c|}{ Tumor size $(\mathrm{cm})$} \\
\hline$<5$ & 17 & 3 & 14 & 0.036 & 0.849 \\
\hline$\geq 5$ & 25 & 5 & 20 & & \\
\hline \multicolumn{6}{|c|}{ Distant metastasis } \\
\hline No & 30 & 2 & 28 & 4.815 & 0.028 \\
\hline Yes & 12 & 6 & 6 & & \\
\hline Total & 42 & 8 & 34 & & \\
\hline
\end{tabular}

correlation was found between LOX-PP expression and AFP level, tumor size, patient gender or age. The cases were then divided into two groups according to the presence of distant metastases. The positive cell incidence of LOX-PP expression was $63.7 \%$ in the specimens with distant metastases, compared to $18.8 \%$ in the specimens without distant metastasis $\left(\chi^{2}=4.815\right.$; $\mathrm{P}=0.028$ ). According to the pathological staging, the cases were divided into three groups: stage I, stage II and stage III. The group with stage II-III showed low positive expression of LOX-PP protein and a significant difference was found among these groups $\left(\chi^{2}=19.216 ; \mathrm{P}<0.001\right)$.

Expression of LOX-PP in SK-Hep-1 and Hep G2 cell lines and the construction of the LOX-PP-overexpressing cell line. Hepatocellular carcinoma SK-Hep-1 and Hep G2 cell lines were used in the present study. Firstly, the endogenous expression of LOX-PP in the SK-Hep-1 and Hep G2 cell lines was evaluated by western blot analysis. As shown in Fig. 1B, a significant lower level of LOX-PP protein expression was observed in the SK-Hep-1 cells $(\mathrm{P}<0.01)$. Thus, SK-Hep-1 cells were chosen as the cells for the infection of the LOX-PP adenovirus (ad-LOX-PP). The adenovirus vector containing the LOX-PP gene (ad-LOX-PP) was constructed for infecting the SK-Hep-1 cells. In pilot studies, the infection efficiency of ad-LOX-PP (at MOI=100) in the SK-Hep-1 cell line was $>90 \%$ as determined by fluorescence microscopy (Fig. 1C). Western 
A

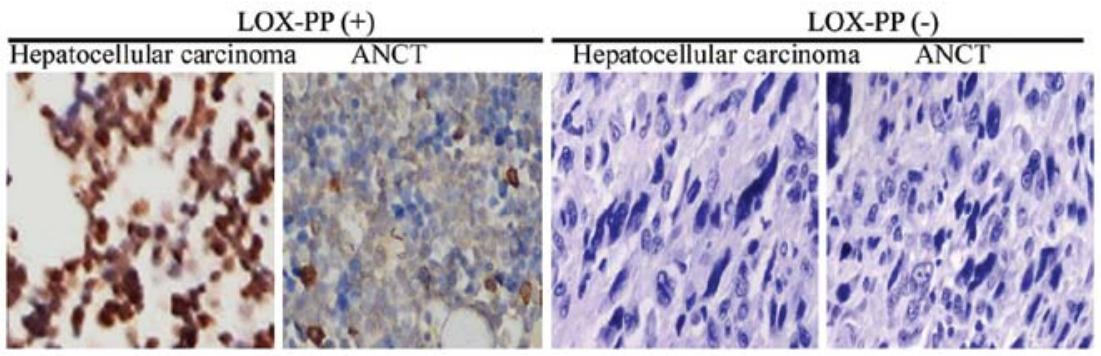

B

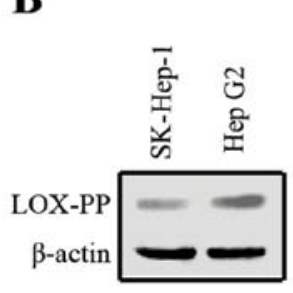

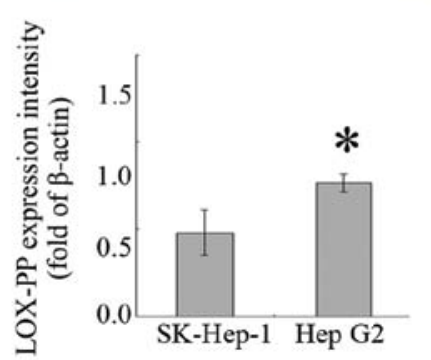

C

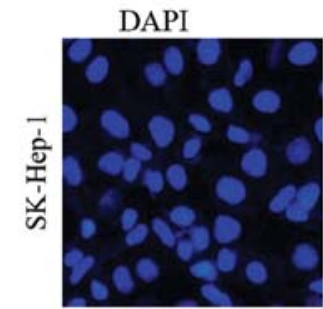

D

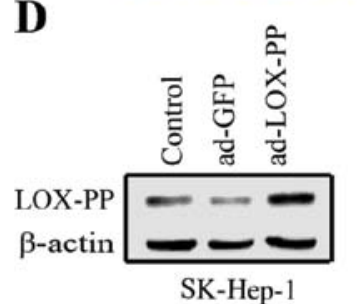

LOX-PP
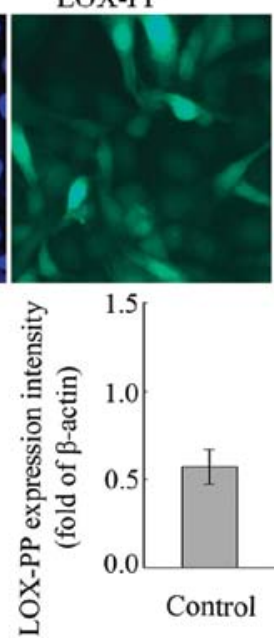

Merge

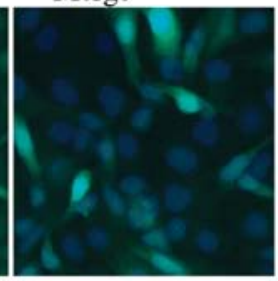

$\mathbf{F}$
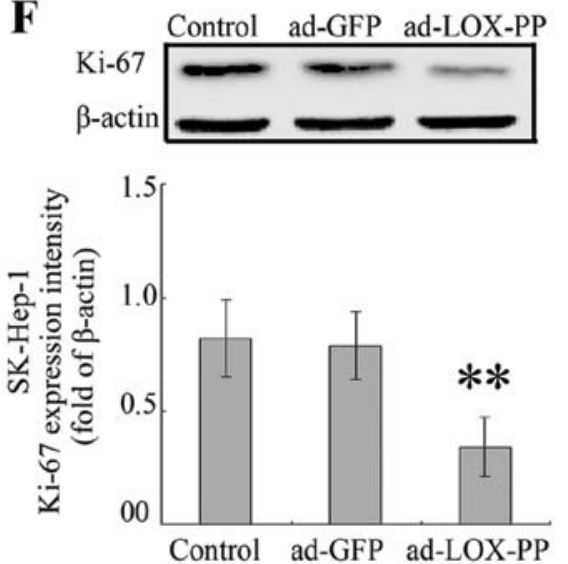

Figure 1. Expression of LOX-PP protein in hepatocellular carcinoma tissues and cell lines and construction of the LOX-PP-overexpressing cell line. (A) Immunostaining of tumors and ANCTs with the LOX-PP antibody. For the negative control, corresponding sections of each tissue were processed in the same manner, omitting the primary antibody (original magnification, x200). (B) The expression of LOX-PP in the SK-Hep-1 and Hep G2 cell lines. The expression level of LOX-PP was significantly lower in the SK-Hep-1 cell line than that in the Hep G2 cell line (" $\mathrm{P}<0.01)$. (C) Adenovirus vector was used to infect the SK-Hep-1 cell line (ad-LOX-PP). The infection efficiency of ad-LOX-PP in the SK-Hep-1 cell line was $>90 \%$ as determined by fluorescence microscopy (original magnification, x200). (D) LOX-PP expression in SK-Hep-1 cells infected with ad-LOX-PP. Western blot assays were performed after 48-h recovery to measure the expression of LOX-PP. A significant increase in LOX-PP expression was observed in the ad-LOX-PP group when compared with that in the ad-GFP and control groups ("P<0.01). (E) CCK-8 assay of LOX-PP-overexpressing cells. LOX-PP significantly reduced the proliferative activities of SK-Hep-1 cells in a time-dependent manner. (F) Western blot assay of Ki-67 expression. Expression of Ki-67 protein was significantly reduced in the ad-LOX-PP group $\left({ }^{* *} \mathrm{P}<0.001\right)$

blot assays were performed after a 48-h recovery to measure the exogenous expression of LOX-PP. An obvious increase in the LOX-PP expression was observed in the ad-LOX-PP group (Fig. 1D) $(\mathrm{P}<0.01)$.

Effect of LOX-PP overexpression on SK-Hep-1 cell proliferation. Abnormal cell proliferation is a hallmark of cancers (17). In order to test the effect of LOX-PP overexpression on SK-Hep-1 cell growth, we investigated the proliferative activities of SK-Hep-1 cells by CCK- 8 assay. As a result, it was found that overexpression of LOX-PP significantly reduced the proliferative activities of SK-Hep-1 cells in a time-dependent manner when compared with that in the ad-GFP and control groups (Fig. 1E). However, no difference was found between the ad-GFP group and the control group.

Ki-67 has been identified as a critical mediator of tumor progression in hepatocellular carcinoma (18) and is widely used to evaluate the outcome of anticancer treatment (19-21). The level of Ki-67 protein was examined by western blot analysis in order to determine whether overexpression of LOX-PP suppressed expression of Ki-67. Our data showed that the expression of Ki-67 protein was significantly decreased in the ad-LOX-PP group when compared with that in the ad-GFP and the control groups $(\mathrm{P}<0.001)$ (Fig. $1 \mathrm{~F})$, suggesting that overexpression of LOX-PP inhibits SK-Hep-1 cell proliferation through downregulation of $\mathrm{Ki}-67$ expression. 

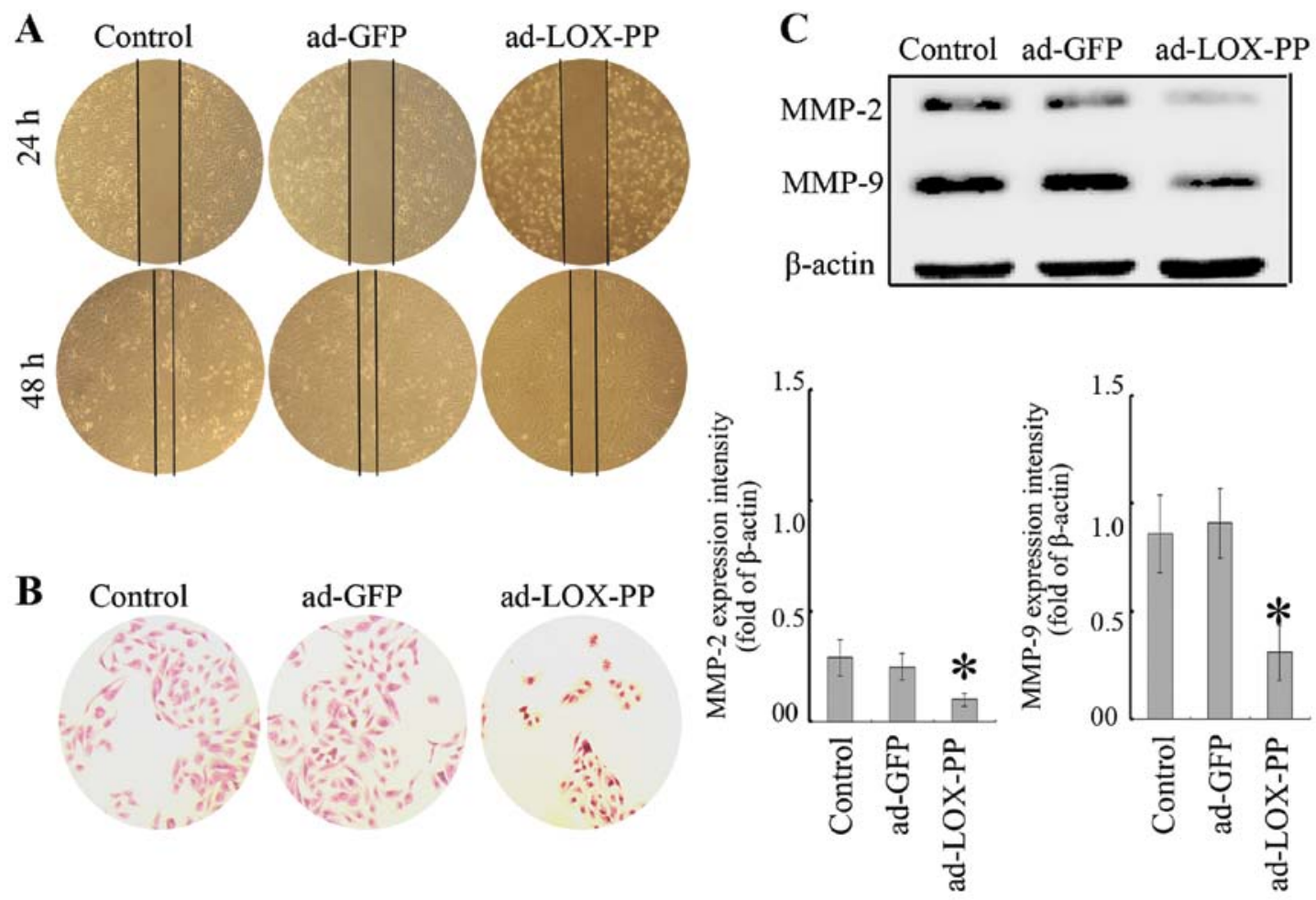

Figure 2. Inhibition of cell migration and invasiveness by LOX-PP in the SK-Hep-1 cell line. (A) Wound-healing assay showed that the migratory capacity of SK-Hep-1 cells in the ad-LOX-PP group was markedly lower than that in the ad-GFP and control groups (original magnification, x200). (B) Transwell invasion assay for examing the transmembrane ability of each group of cells. The quantitative results showed a reduced ability in the ad-LOX-PP group (original magnification, x200). (C) Western blot assay was performed to examine the effect of LOX-PP overexpression on MMP-2 and MMP-9 expression. The levels of MMP-9 and MMP-2 proteins were significantly decreased in the ad-LOX-PP group when compared with levels in the ad-GFP and control groups ("P<0.01).

Effect of LOX-PP overexpression on SK-Hep-1 cell invasion and metastasis. Wound-healing and Transwell assays were carried out to determine the effect of LOX-PP overexpression on SK-Hep-1 cell invasion and metastasis. As shown in Fig. 2A, wound-healing results showed that the migratory capacity of SK-Hep-1 cells in the ad-LOX-PP group was markedly reduced. Yet, no significant differences in the migrative capacity were found between the ad-GFP and the control groups. Representative micrographs of Transwell filters are shown in Fig. 2B. The invasive activity of SK-Hep-1 cells was decreased in the ad-LOX-PP group when compared with that in the ad-GFP and control groups $(\mathrm{P}<0.01)$.

To investigate the possible mechanisms of LOX-PP on invasion and metastasis (22-24), using western blot analysis we detected expression of the MMP-9 and MMP-2 which are critical regulators of tumor invasion, progression and metastasis in multiple cancer types. As shown in Fig. 2C, MMP-9 and MMP-2 expression was significantly reduced in the ad-LOX-PP group when compared with that in the ad-GFP and control groups $(\mathrm{P}<0.01)$, indicating that LOX-PP overexpression may inhibit SK-Hep-1 cell invasion and metastasis through downregulation of MMP-9 and MMP-2 expression.

Effects of LOX-PP overexpression on SK-Hep-1 cell apoptosis and cycle distribution. To determine whether LOX-PP overexpression affects SK-Hep-1 cell apoptosis, flow cytometric analysis with Annexin V-PE/PI staining was performed. The results demonstrated that the rate of apoptosis of SK-Hep-1 cells in the ad-LOX-PP group was distinctly higher than that in the ad-GFP and control groups $(\mathrm{P}<0.01)$ (Fig. 3A). No significant difference in the rate of apoptosis was found between the ad-GFP and the control groups. Additionally, the SK-Hep-1 cell cycle distribution was also analyzed and cell cycle kinetics showed that the G0/G1 phase fraction was increased in the ad-LOX-PP group, while the $\mathrm{S}$ phase fraction was decreased (Fig. 3B). In contrast, cell cycle characteristics of GFP-negative cells were similar to those of the untransfected counterparts.

Cyclin D1 is responsible for cell cycle progression during the $\mathrm{G} 1$ phase (25), and cyclin E functions in the progression of cells from late G1 to early S phase (26). Thus, western blot assay was performed to investigate the effect of LOX-PP overexpression on the endogenous expression of cyclin $\mathrm{E}$ and cyclin D1 proteins. As shown in Fig. 3C, the expression of cyclin $\mathrm{E}$ was significantly decreased in the ad-LOX-PP group compared with that in the ad-GFP and control groups (each $\mathrm{P}<0.01$ ), indicating that overexpression of LOX-PP induces cell cycle arrest through downregulation of cyclin E expression. No significant differences in cyclin D1 were found among the LOX-PP, ad-GFP and control groups.

Overexpression of LOX-PP and its effect on PIBK/AKT and $M A P K$ expression. Mitogen-activated protein kinase (MAPK) and phosphoinositide 3-kinase (PI3K) pathways are at the center of signaling networks that govern proliferation, differentiation and cell survival, therefore, dysfunction is associated with tumorigenesis $(19,27)$. This could be activated by a variety 
$\mathbf{A}$

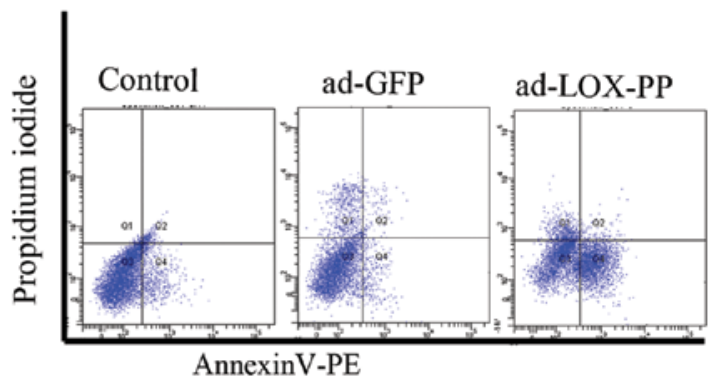

B
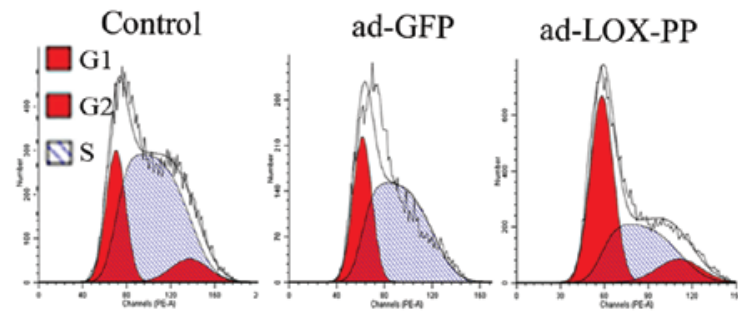

C
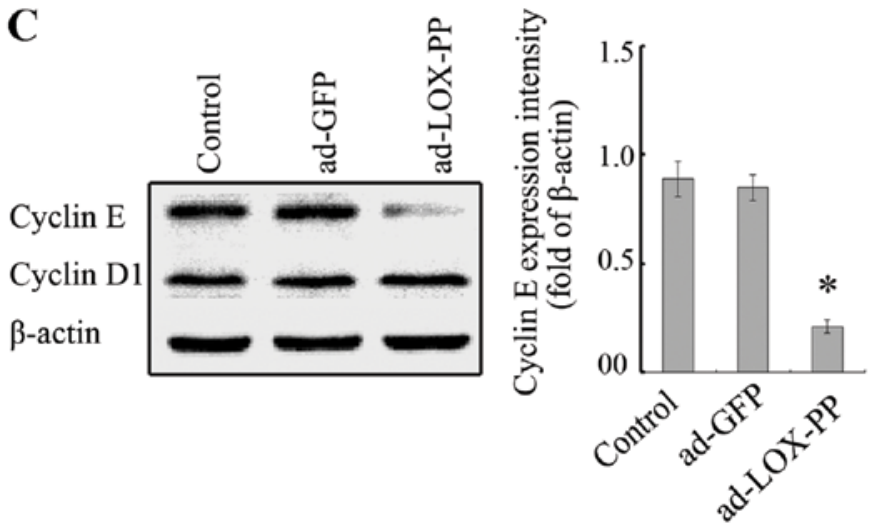
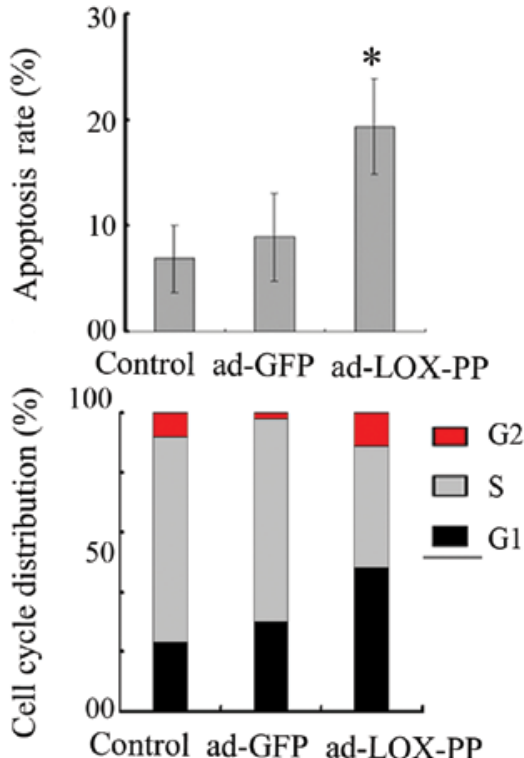

Control ad-GFP ad-LOX-PP

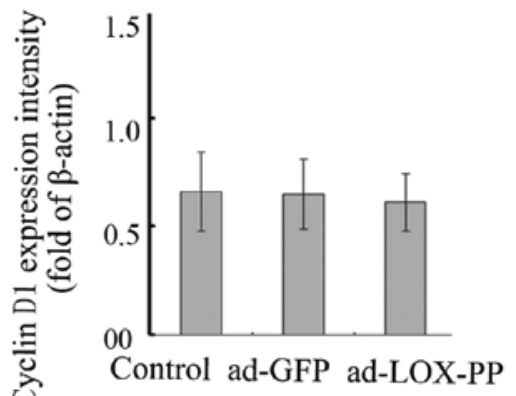

Figure 3. Effect of LOX-PP on apoptosis and cell cycle distribution in the SK-Hep-1 cell line. (A) At the end of the incubation period and $48 \mathrm{~h}$ after transfection, the cells were stained with Annexin V-PE/PI, and the rate of apoptosis was analyzed by flow cytometry. The percentage of apoptotic cells was increased in the ad-LOX-PP group. (B) Cell cycle distribution was analyzed by flow cytometric analysis. Overexpression of LOX-PP caused G0/G1 arrest and a significant decrease in the S phase population. (C) Western blot assay for cyclin E and cyclin D1 expression. Expression of cyclin E was significantly inhibited in the ad-LOX-PP group when compared to that in the ad-GFP and control groups ( $\mathrm{P}<0.01)$. No significant differences in cyclin D1 were found among the LOX-PP, ad-GFP and control groups.

of signals, including growth factors, inflammatory cytokines, oxidative stress, and carcinogens (28) and participate in tumor angiogenesis (29).

We next evaluated the expression levels of the markers of the PI3K and MAPK signaling pathway, including AKT, p-AKT, PI3K, p-PI3K, JNK, p-JNK, P38, p-P38, p-ERK and ERK by western blot analysis. The expression levels of AKT, p-AKT, PI3K, p-PI3K, JNK, p-JNK, P38 and p-P38, were not significantly different among the LOX-PP, ad-GFP and control groups (Fig. 4A). However, an obvious decreased expression of p-ERK was observed in the ad-LOX-PP group when compared with its level in the ad-GFP and control groups (each $\mathrm{P}<0.01$ ). Our data suggest that LOX-PP overexpression induces apoptosis through dephosphorylation of ERK.

\section{Discussion}

Cancer invasion and metastasis are complex processes involving an increase in cell motility, vascular invasion and digestion of extracellular matrix. Many mechanisms regulate these processes, and one technique with which to identify the controlling genes involved in these processes is to compare the gene expression between tumor tissue and ANCTs $(30,31)$. LOX-PP is a multifunctional protein with intranuclear and extracellular functions. An antitumor effect of LOX-PP was reported in breast (7), pancreas (12), lung (32), prostate (8), gastrointestinal (33) and many other organs. Overexpression of LOX-PP was found to inhibit the growth of a pre-existing breast cancer xenograft (7) and to attenuate the migratory phenotype of lung cancer cells (34).

In the present study, compared with the ANCTs, we identified LOX-PP as a low expression gene in hepatocellular carcinoma by IHC analysis. The relationships between LOX-PP expression and various clinical features were also analyzed. The results showed that low expression of LOX-PP was an indicator of poor prognosis and advanced stage tumors. These above observations indicate that LOX-PP is commonly downregulated in cancer and may exert an antitumor effect. Therefore, the role of LOX-PP in hepatocellular carcinoma was further investigated in vitro.

Overexpression of LOX-PP was successfully induced in SK-Hep-1 cells, and an obvious increase in LOX expression 
A

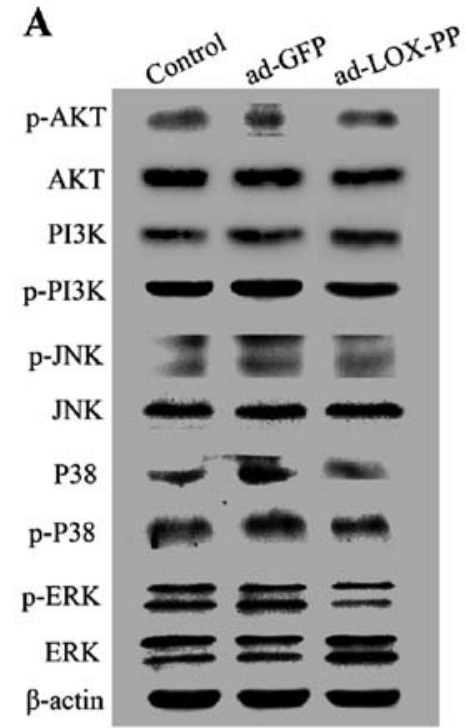

B

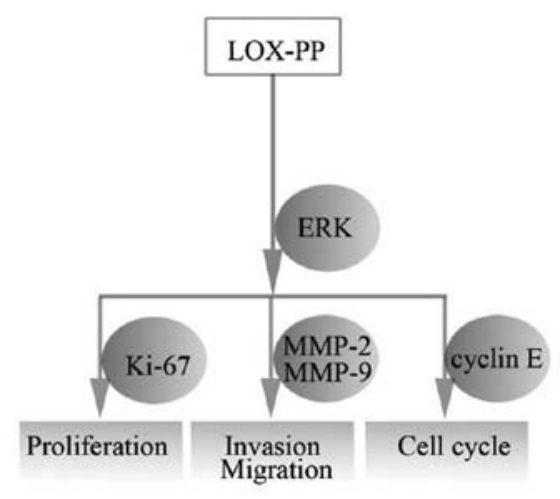

Figure 4. Overexpression of LOX-PP and its effect on the PI3K/AKT and MAPK signaling pathways. (A) Western blot assay was performed following a 48-h recovery to determine the expression of AKT, p-AKT, PI3K, p-PI3K, JNK, p-JNK, P38 and p-P38. An obvious decrease in p-ERK expression was observed in the ad-LOX-PP group when compared with that in the ad-GFP and control groups ("P<0.01). (B) Schematic representation of the proposed mechanism of LOX-PP -mediated tumor inhibition in SK-Hep-1 cells.

was observed in the ad-LOX group. The present study showed that endogenous overexpression of LOX-PP significantly inhibited the proliferative activities. Consistent with our finding, a previous study showed that LOX-PP inhibited the growth of a prostate cancer cell line through inhibition of ERK1/2 and AKT signaling (8). In contrast, our results showed that the possible mechanism was through downregulation of Ki-67 expression. Furthermore, overexpression of LOX-PP also resulted in cell cycle arrest and enhanced apoptosis. A previous study reported that LOX-PP reduced the growth of MIA PaCa-2 pancreatic cancer cells via downregulation of cyclin D1 (12), In contrast, we found that LOX-PP suppressed cell proliferation via downregulation of cyclin E. More importantly, overexpression of LOX-PP also inhibited invasion and migration of hepatocellular carcinoma cells evidenced by the Transwell migration and scratch wound assays. Subsequently, we further tested the important signaling pathways involved in cell migration. Out data showed that the reduced invasiveness was mostly attributed to a decrease in MMP-2 and MMP-9 expression. The role of LOX-PP was consistent with Hurtado et al (9) who reported that overexpression of LOX-PP inhibited proliferation of primary rat aorta smooth muscle cells via downregulation of MMP-9.

MAPK/ERK is at the center of signaling networks that govern proliferation, differentiation and cell survival, and presents one of the major pathways for regulating malignant progression of various types of tumors. Thus, the MAPK/ERK pathway may be involved in the possible mechanism by which LOX-PP affects proliferation, migration and the cell cycle of hepatocellular carcinoma cells. The present study revealed a marked reduction in p-ERK expression, with a concomitant decrease in cell proliferative activitiy and migration capacity. To the best of our knowledge, this is the first report elucidating the role of LOX-PP in the growth and migration of hepatocellular carcinoma cells and the proposed mechanism as shown in Fig. 4B. However, tumor initiation and progression are closely associated with cell type and microenvironment of adjacent tissues. Therefore, the present study provides preliminary evidence, which requires further research using more cell lines to explore the specific mechanisms $(35,36)$.

In conclusion, the present study revealed that the enhanced expression level of LOX-PP by ad-LOX-PP transfection exerted inhibitory effects on the growth and migration of hepatocellular carcinoma cells possibly via the blockade of MAPK/ERK signaling. LOX-PP as a tumor suppressor may provide a novel approach for human hepatocellular carcinoma treatment.

\section{References}

1. El-Serag HB and Rudolph KL: Hepatocellular carcinoma: epidemiology and molecular carcinogenesis. Gastroenterology 132: 2557-2576, 2007.

2. Hsu HC, Huang AM, Lai PL, et al: Genetic alterations at the splice junction of p53 gene in human hepatocellular carcinoma. Hepatology 19: 122-128, 1994.

3. Hsu HC, Jeng YM, Mao TL, et al: $\beta$-catenin mutations are associated with a subset of low-stage hepatocellular carcinoma negative for hepatitis B virus and with favorable prognosis. Am J Pathol 157: 763-770, 2000.

4. Yuan JM, Govindarajan S, Ross RK, et al: Chronic infection with hepatitis $G$ virus in relation to hepatocellular carcinoma among non-Asians in Los Angeles County, California. Cancer 86: 936-943, 1999.

5. Kagan HM and Trackman PC: Properties and function of lysyl oxidase. Am J Respir Cell Mol Biol 5: 206-210, 1991.

6. Kim YM, Kim EC and Kim Y: The human lysyl oxidase-like 2 protein functions as an amine oxidase toward collagen and elastin. Mol Biol Rep 38: 145-149, 2011.

7. Bais MV, Nugent MA, Stephens DN, et al: Recombinant lysyl oxidase propeptide protein inhibits growth and promotes apoptosis of pre-existing murine breast cancer xenografts. PLoS One 7: e31188, 2012.

8. Palamakumbura AH, Vora SR, Nugent MA, et al: Lysyl oxidase propeptide inhibits prostate cancer cell growth by mechanisms that target FGF-2-cell binding and signaling. Oncogene 28: 3390-3400, 2009.

9. Hurtado PA, Vora S, Sume SS, et al: Lysyl oxidase propeptide inhibits smooth muscle cell signaling and proliferation. Biochem Biophys Res Commun 366: 156-161, 2008. 
10. Min C, Kirsch KH, Zhao Y, et al: The tumor suppressor activity of the lysyl oxidase propeptide reverses the invasive phenotype of Her-2/neu-driven breast cancer. Cancer Res 67: 1105-1112, 2007.

11. Palamakumbura AH, Jeay S, Guo Y, et al: The propeptide domain of lysyl oxidase induces phenotypic reversion of ras-transformed cells. J Biol Chem 279: 40593-40600, 2004.

12. Min C, Zhao Y, Romagnoli M, et al: Lysyl oxidase propeptide sensitizes pancreatic and breast cancer cells to doxorubicininduced apoptosis. J Cell Biochem 111: 1160-1168, 2010.

13. Barker HE, Cox TR and Erler JT: The rationale for targeting the LOX family in cancer. Nat Rev Cancer 12: 540-552, 2012.

14. Edmondson HA and Steiner PE: Primary carcinoma of the liver: a study of 100 cases among 48,900 necropsies. Cancer 7: 462-503, 1954

15. Yang YH, Chen K, Li B, et al: Estradiol inhibits osteoblast apoptosis via promotion of autophagy through the ER-ERKmTOR pathway. Apoptosis 18: 1363-1375, 2013.

16. Chiu CC, Lin $\mathrm{CH}$ and Fang K: Etoposide (VP-16) sensitizes p53-deficient human non-small cell lung cancer cells to caspase-7-mediated apoptosis. Apoptosis 10: 643-650, 2005.

17. Novello C, Pazzaglia L, Cingolani C, et al: miRNA expression profile in human osteosarcoma: Role of miR-1 and miR-133b in proliferation and cell cycle control. Int J Oncol 42: 667-675, 2013.

18. Aigelsreiter A, Ress AL, Bettermann K, et al: Low expression of the putative tumour suppressor spinophilin is associated with higher proliferative activity and poor prognosis in patients with hepatocellular carcinoma. Br J Cancer 108: 1830-1837, 2013.

19. Li B, Yang Y, Jiang S, et al: Adenovirus-mediated overexpression of BMP-9 inhibits human osteosarcoma cell growth and migration through downregulation of the PI3K/AKT pathway. Int J Oncol 41: 1809-1819, 2012.

20. Liu ZL, Wang G, Peng AF, et al: Fatty acid synthase expression in osteosarcoma and its correlation with pulmonary metastasis. Oncol Lett 4: 878-882, 2012.

21. Thompson L, Wang S, Tawfik O, et al: Effect of 25-hydroxyvitamin $\mathrm{D}_{3}$ and $1 \alpha, 25$ dihydroxyvitamin $\mathrm{D}_{3}$ on differentiation and apoptosis of human osteosarcoma cell lines. J Orthop Res 30: 831-844, 2012.

22. Kamei S, Sakayama K, Tamashiro S, et al: Ketoprofen in topical formulation decreases the matrix metalloproteinase-2 expression and pulmonary metastatic incidence in nude mice with osteosarcoma. J Orthop Res 27: 909-915, 2009.

23. Korpi JT, Hagstrom J, Lehtonen N, et al: Expression of matrix metalloproteinases-2, $-8,-13,-26$, and tissue inhibitors of metalloproteinase-1 in human osteosarcoma. Surg Oncol 20: e18-22, 2011.
24. Zhang Y, Song L, Cai L, et al: Effects of baicalein on apoptosis, cell cycle arrest, migration and invasion of osteosarcoma cells. Food Chem Toxicol 53C: 325-333, 2012.

25. Gu X, Xing L, Shi G, et al: The circadian mutation $P E R 2^{S 662 G}$ is linked to cell cycle progression and tumorigenesis. Cell Death Differ 19: 397-405, 2012.

26. Oka K, Ohya-Shimada W, Mizuno S, et al: Up-regulation of cyclin- $E_{1}$ via proline-mTOR pathway is responsible for HGF-mediated $\mathrm{G}_{1} / \mathrm{S}$ progression in the primary culture of rat hepatocytes. Biochem Biophys Res Commun 435: 120-125, 2013.

27. Rasmussen $\mathrm{N}$ and Rathmell WK: Looking beyond inhibition of VEGF/mTOR: emerging targets for renal cell carcinoma drug development. Curr Clin Pharmacol 6: 199-206, 2011.

28. Van Waes C: Nuclear factor- $\kappa B$ in development, prevention, and therapy of cancer. Clin Cancer Res 13: 1076-1082, 2007.

29. Peng L, Liu A, Shen Y, et al: Antitumor and anti-angiogenesis effects of thymoquinone on osteosarcoma through the NF- $\mathrm{BB}$ pathway. Oncol Rep 29: 571-578, 2013.

30. Jeng YM, Chang CC, Hu FC, et al: RNA-binding protein insulin-like growth factor II mRNA-binding protein 3 expression promotes tumor invasion and predicts early recurrence and poor prognosis in hepatocellular carcinoma. Hepatology 48: 1118-1127, 2008.

31. Chen YL, Wang TH, Hsu HC, et al: Overexpression of CTHRC1 in hepatocellular carcinoma promotes tumor invasion and predicts poor prognosis. PLoS One 8: e70324, 2013.

32. Sanchez-Morgan N, Kirsch KH, Trackman PC, et al: The lysyl oxidase propeptide interacts with the receptor-type protein tyrosine phosphatase kappa and inhibits $\beta$-catenin transcriptional activity in lung cancer cells. Mol Cell Biol 31: 3286-3297, 2011.

33. Yoon JH, Park JK, Kang YH, et al: Lysyl oxidase G473A polymorphism is closely associated with susceptibility to gastric cancer in a South Korean population. APMIS 119: 762-768, 2011.

34. Yu Z, Sato S, Trackman PC, et al: Blimp1 activation by AP-1 in human lung cancer cells promotes a migratory phenotype and is inhibited by the lysyl oxidase propeptide. PLoS One 7: e33287, 2012.

35. Barcellos-Hoff MH and Ravani SA: Irradiated mammary gland stroma promotes the expression of tumorigenic potential by unirradiated epithelial cells. Cancer Res 60: 1254-1260, 2000.

36. Wiseman BS and Werb Z: Stromal effects on mammary gland development and breast cancer. Science 296: 1046-1049, 2002. 